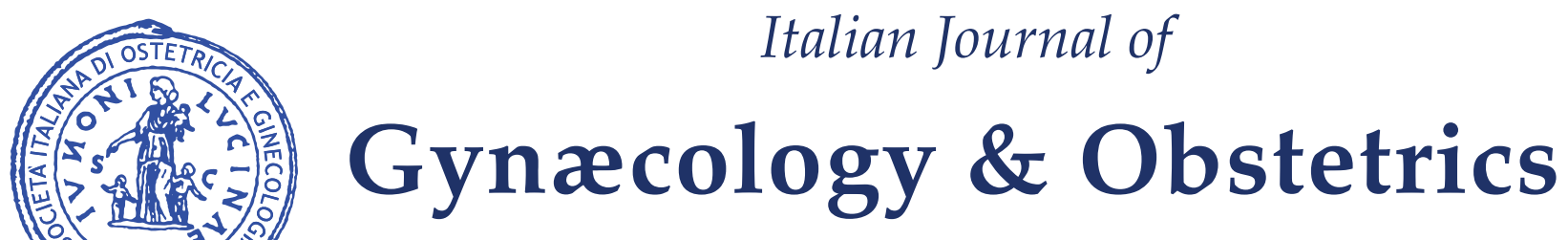

September 2020 - Vol.32 - N. 3 - Quarterly - ISSN 2385 - 0868

\title{
Sterility Special Interest Group position paper on ART treatments and COVID-19 pandemic
}

\author{
C. Alviggi ${ }^{1,2}$, A. Borini ${ }^{2}$, M. Costa ${ }^{3}$, G. D' Amato $^{4}$, L. Gianaroli ${ }^{5}$, N. Colacurci ${ }^{6}$, on behalf of the Sterility \\ Special Interest Group (GISS) 7 of the Italian Society of Gynaecology and Obstetrics (SIGO) and its \\ federated associations
}

\begin{abstract}
${ }^{1}$ Department of Neuroscience, Reproductive Sciences and Odontostomatology, School of Medicine, Federico II University, Naples, Italy

${ }^{2}$ Baby Family and Fertility Center, Centre for Reproductive Health, Bologna, Italy

${ }^{3}$ Reproductive Medicine Department, International Evangelic Hospital, Genoa, Italy

${ }^{4}$ Asl Bari, DPT Maternal and Child Health, Reproductive and IVF Unit, PTA F Jaia, 70014, Conversano, Bari, Italy

${ }^{5}$ Reproductive Medicine Unit, S.I.S.Me.R., Bologna, Italy

${ }^{6}$ Department of Woman, Child and General and Specialized Surgery, Obstetrics and Gynecology Unit, University of Campania Luigi Vanvitelli, Naples, Italy

${ }^{7}$ Members of the Sterility Special Interest Group (GISS), in alphabetic order: S. Ajossa, C. Alviggi, P. Anserini, M. Bertoli, A. Borini, G. Cau, N. Colacurci, M. Costa, G. D'Amato, G. De Placido, C. De Stefano, L. Gianaroli, P. E. Levi Setti, L. Mencaglia, R. Palermo, A. Perino, E. Porcu, S. Schettini, F. M. Ubaldi

All authors equally contributed to the manuscript and are indicated in rigorous alphabetic order, having as last Nicola Colacurci as Coordinator of the Sterility Special Interest Group
\end{abstract}

\section{ABSTRACT}

COVID-19 pandemic caused a huge overload of healthcare systems worldwide. For such reason, in a preventive manner, governments and scientific societies recommended to stop any elective medical or surgical treatment in order to reduce the eventual burden on hospitals. Fertility treatments have since then been reserved only to urgent cases as oncologic patients asking for fertility preservation. However, the relevance of such policy on natality rate and on ovarian aging has soon induced the main scientific societies to ask for a fast return to action, considering that infertility should be treated as an urgent condition. In this regard, phase 2 recommendations have been issued to ensure working requirements to be settled in the safest way possible. Therefore, the Special Interest Group on Sterility (GISS) of the Italian Society of Gynecology and Obstetrics (SIGO) and its federates released their guidelines as Italy has been one of the first Western countries to face the phase 2 restart of treatments.

\section{SOMMARIO}

La pandemia dovuta al COVID-19 ha causato un imponente sovraccarico dei sistemi sanitari di tutto il mondo. Per questa ragione, in maniera preventiva, i governi e le società scientifiche hanno raccomandato di interrompere ogni trattamento medico o chirurgico elettivo per ridurre l'eventuale peso sugli ospedali. Da quel momento, i trattamenti per la fertilità sono stati riservati solo a casi urgenti, come pazienti oncologici che richiedevano procedure di preservazione della fertilità. Pertanto, la rilevanza di questa politica sul tasso di natalità e sull'invecchiamento ovarico ha presto indotto le principali società scientifiche a spingere per un rapido ritorno all'attività, considerando che l'infertilità dovrebbe essere trattata come una condizione urgente. A tal proposito, sono state rilasciate le raccomandazioni di fase 2 per assicurare che i requisiti lavorativi fossero organizzati nella maniera più sicura possibile. Quindi, il Gruppo di Interesse Speciale per la Sterilità (GISS) della Società Italiana di Ginecologia e Ostetricia (SIGO) e le sue federate hanno rilasciato le proprie linee guida, dato che l'Italia è stata una delle prime nazioni occidentali ad affrontare l'inizio dei trattamenti in fase 2. 
Corresponding Author: Carlo Alviggi

E-mail: alviggi@unina.it

Copyright 2020

DOI: 10.36129 / jog.32.03.01

\section{INTRODUCTION}

The Sterility Special Interest Group (GISS) of the Italian Society of Gynaecology and Obstetrics (SIGO) and its federated associations (AOGOI Italian Hospital Obstetricians and Gynaecologists Association), (AGUI - Italian University Gynaecologists Association), (AGITE - Community Gynaecologists Association), wishes to contribute to the establishment of the methods, times and conditions by which the ART (Assisted Reproductive Technology) Centres will be able to operate after the general lockdown phase (phase 1), guaranteeing the highest possible degree of safety for the couples and operators (phase 2). With regard to ART treatments, on 18th March, our GISS and the entire SIGO made the following statements:

1. the movement of people and their access to the clinical facilities must be avoided to reduce the risk of contagion. The exponential increase in the number of COVID-19 cases, in spite of the precautions taken in application of the Government decrees (Prime Minister decrees DPCM dated 8, 9 and 11 March 2020), increases the risk of contamination of infertile patients and the personnel of the assisted reproduction centers;

2. it is ethically important to avoid creating any situation that may result in patients having to go to First aid departments or be admitted to hospital after an ART cycle. To reduce the risks of having to occupy beds in hospital, operating rooms or intensive care units, as a result of complications related to the performance of assisted conception programmes, is a duty towards the population and colleagues involved in a fight at the limit of human and economic resources;

3. in conclusion, the GISS feels that the only exceptions to the suspension of activities are represented by stimulation cycles already started and fertility cryopreservation procedures in cancer patients, which will be guaranteed by the designated facilities, considering their urgency and undeferrability.
Key words:

COVID-19; assisted reproduction technologies; pandemic; in-vitro fertilization; poor prognosis; position paper.

Our suggestions for avoiding the spread of contagion were in line with the recommendations of the main international scientific societies, all of which have underlined the need to refrain from initiating assisted reproduction treatments during the rise to the peak of infection to avoid spreading contagion. All the societies agreed that the treatments should be postponed to the fall from the peak, forecast over a period of time of no more than two or three months (1-3).

The Italian Prime Minister himself has tried to divide the long period for which the coronavirus will be present in Italy into phases: a first phase (the current lockdown phase), a second phase (which we will enter when the number of people testing positive decreases with a consequent reduction of the number of deaths) and a third phase (the return to normality with large groups of immunized people).

A premise to every subsequent organizational phase is the fact that, according to the currently available literature and also the large number of cases collected from our hospitals, there seems to be no or negligible vertical transmission of COVID-19 from mother to child (4). In fact, most mothers found to be COVID-19 positive during delivery have given birth to negative babies. Only three cases of babies born positive have been reported so far.

No health authority of any country has issued indications or recommendations to the population discouraging women from becoming pregnant in this period.

For this reason, we believe that, as long as there is no scientific evidence that advise against starting a pregnancy, the need to suspend ART treatments is technically and scientifically unfounded.

The purpose of this document is to prepare for the complete resumption of the reproductive medicine activities following the start of the second phase, to eliminate the latency times between the start of the second phase and the resumption of the clinical and care ART activities at full rhythm. This document is therefore aimed at defining the 
path to be followed to promptly resume the treatments for our patients as soon as the start of the second phase is officialised.

Sterility treatment is considered a right of couples with reproduction problems; this is underlined several times in law no. 40, confirmed by the Constitutional Court in its changes to the law and, finally, implemented by the Ministry of Health as ART treatments were added to the List of Minimum Healthcare Provisions (5).

Our country has an infertile population among the oldest in the Western world (6), with the first treatments administered to couples in which the women have an average age of 37 years and, in a third of the cases, the woman is aged over 40 years. Excessive delays in treatment may significantly reduce the possibility of success for these couples. It is therefore necessary to resume these treatments as soon as the general lockdown phase is terminated, bearing in mind that every month of inactivity in Italy means about 7,500-8,000 fewer treatments administered with a potential monthly loss of about 1500 births (7).

When the second phase begins, one of the most important reasons for deciding to suspend the activity during the first phase of the epidemic, that is the need to avoid overloading the emergency health facilities with possible problems related to the performance of ART cycles, will no longer be valid. In fact, in the second phase, there will be no overcrowding of the emergency departments so all other healthcare activities will return to their normal rhythm and the ART activity must also be resumed.

In addition, it should be taken into consideration that the ART centres handle their procedures autonomously, and with an extremely low risk of infection, in that the authorized centres are classified as Tissue establishments, and thus have certified procedures and facilities. In addition, the surgical complications that may occur and require a visit to a First Aid department are extremely limited: $0.13 \%$ of haemoperitoneum after the oocyte retrieval, $0.04 \%$ of pelvic abscesses or infections and $0.35 \%$ of hyperstimulation syndromes (2019 Ministry of Health report to the Parliament) (8).

The procedure that we recommend be followed at the ART centres for the resumption of activities is set out here below.

\section{RECOMMENDATIONS ON THE SIMULTANEOUS MANAGEMENT OF THE ACCESS AND CIRCULATION OF PATIENTS AT THE FACILITIES WHERE THE ART PROCEDURES ARE CARRIED OUT}

\section{Remote management of patients}

In the new scenario, the occasions on which the couples have to leave their workplaces will have to be reduced to a minimum, both due to the predictable difficulties of the Economic System but above all to reduce the risk of spreading the virus by limiting the circulation of the couples at the health facilities.

In this sense, we agree with the fifth statement made recently by the ASRM (effective from 30 March - 13 April 2020)(9), which identifies the use of TeleMedicine (TM) tool as a solution to the need to guarantee the greatest possible "distancing of the patients from the operators".

The need to use this tool has been confirmed on a national level by members of the GISS $(10,11)$.

TM should be used for the following services:

- remote consultation;

- remote diagnostics;

- remote monitoring.

The supply of these services by "e-health" would reduce to a minimum the time spent by patients in waiting rooms and outpatient departments.

As the data transferred by TM are extremely sensitive, the use of certified platforms for the production and protection of this data is recommended.

This innovative approach requires institutional recognition (establishment of an Operating Procedure and Public accreditation).

Its efficacy can be assessed by adopting the NIMM (NHS Infrastructure Maturity Model) adopted in Great Britain.

It should be pointed out that the TM tool has already been indicated in the 2017 Guideline elaborated by the Ministry of Health, which indicated "e-health" as one of the priority areas for the development of interventional policies in the healthcare sector.

The SIGO is ready to stand by the institutions in designing a certified and recognized method on an institutional level.

As legislative decree no. 40/2004 is still in force, some provisions laid down in the law should be 
adapted to this innovative procedure, making them "not face to face". This would not interfere with the relationship of trust between the couple and the Centre's team. On the contrary, the use of TM would enable medical consulting and checks of the progress of the treatments to be distinguished better and conducted at a distance, also through interviews with the nursing support staff who could deliver documents certified by the medical team.

\section{Access to treatments}

Every ART centre is a tissue establishment that operates in a protected environment and setting and should constantly safeguard both the patients and the operators. Symptomatic SARS-COV-2-positive patients with a COVID-19 diagnosis must be excluded from all ART treatments. Similarly, infected operators or operators suspected of carrying the infection must be isolated from the ART centre.

Treatment should not be given to patients at a high risk of COVID-19 infection/complications due to existing clinical conditions, for example, kidney disease, diabetes, hypertension, liver disease, heart disease and all disorders that cause immune impairment, such as AIDS or malnutrition.

Prior to the ART pathway, the patient's medical history should be drawn up with particular reference to the risk of exposure, and a preliminary clinical assessment should be made. To do this, an initial telephonic triage form should be filled in (figure 1). This approach would enable the healthcare operator to formulate the questions appropriately and assess more directly the adequacy of the answers given. The triage questionnaire should, in any case, be sent also by e-mail to enable the couple to fill in, sign and return it. The best time to give the questionnaire to the patient is 7-10 days before the start of the ovarian stimulation cycle or, for Frozen Embryo Transfer (FET), the start of the endometrial preparation cycle.

The contents of the triage form will be updated periodically on the basis of the information that becomes available about the spread of the virus, the mobility directives and the latest provisions laid down by the competent bodies.

The transmission of the triage form 7-10 days before the presumed date of the start of controlled ovarian stimulation (COS) not only guarantees a preliminary assessment of the couple's eligibility for the ART cycle, it also provides two fundamental advantages: a) the possibility of offering extensive and exhaustive counselling on the preventive measures to be adopted in the period immediately before the ART procedure and for its entire duration;

b) the availability of a window period in which to stratify the patient's risk status better, that is, to observe the onset of any symptoms (if the medical history is positive for a risk of exposure) and / or carry out any necessary diagnostic tests, such as the rapid immunoglobulin test (which can be repeated 7 days after resulting negative, if appropriate) or the swab (in patients with significant symptoms).

On the basis of the pre-ART triage result, various scenarios are possible (figure 2).

\section{Scenario 1}

Patient and partner with no symptoms and a negative triage result. Counselling will be given on preventing the risk of exposure and the ART procedure will be carried out using the standard methods used at the health facility.

\section{Scenario 2}

Patient and/or partner with positive triage result but only a risk of exposure and/or mild non-specific symptoms.

In this scenario, a reassessment will have to be made by triage on the second day of menstruation or the day planned for the start of controlled ovarian stimulation. In the interval of 7-10 days between the two triage procedures, infectious disease consulting can be requested or appropriate diagnostic tests can be ordered directly.

If, during the window period, no symptoms develop and/or the diagnostic tests or consultant examinations conducted do not reveal signs of recent infection, the situation would be equivalent to "scenario 1" and the couple would start ART treatment in accordance with the standard procedures.

\section{ACCESS TO TREATMENTS}

First triage by phone: $7-10$ days before the initiation of COS or endometrial preparation for ET

Second triage: on the second day of menstrual cycle, or first day of COS or endometrial preparation for ET

Additional check point: on the day of ovulation triggering or first day of progesterone administration in endometrial preparation for ET cycle

Figure 1. Check points at crucial steps of IVF programs COS, controlled ovarian stimulation; ET, embryo transfer. 
The couple should be informed of the risk of a cancellation of the cycle if evidence of an infection in progress appears.

\section{Scenario 3}

Patient and/or partner with manifest symptoms and/or a positive test. Under such circumstances, ART treatment must be deferred, and the couple must be directed towards an appropriate diagnostic pathway at the competent facilities. All couples that passed the initial triage should be subjected to another check at the end of the stimulation cycle, preferably on the day of ovulation triggering or at the start of supplementation with progesterone in the case of endometrial preparation for FET. The aim is to detect any risk factors and / or symptoms that appear during the stimulation or endometrial preparation procedure. This assessment can be made using a triage form similar to the previous one. Even in this case, the 3 scenarios described above could arise.

\section{Scenario 1}

Patient and partner with no symptoms and a negative pre-triggering/FET triage result.

Oocyte retrieval and the frozen embryo transfer will be carried out regularly unless significant symptoms appear after the questionnaire has been given.

\section{Scenario 2}

Patient and/or partner with positive pre-triggering/FET triage result, even if only with a risk of exposure and/or mild non-specific symptoms. In these cases, a rapid test should be carried out, which will be decisive as to whether the cycles can be continued or have to be suspended.

\section{Scenario 3}

Patient and/or partner with manifestly positive pre-triggering/FET triage result. Under such circumstances, oocyte retrieval or the frozen embryo transfer will have to be deferred, and the couple will have to be directed towards an appropriate diagnostic pathway (infectious disease/pulmonary consulting examination and diagnostic laboratory tests). The only possible exception is for oocyte retrieval in patients with a high risk of developing severe ovarian hyperstimulation (figure 3 ).

For deferred treatments, the possibility of carrying out the ART procedure in the subsequent cycles will be considered, upon exhibition of documenta- tion certifying the patient's negative infection test result.

\section{Management of access to the Centre and organiza- tion of the various phases}

\section{Premises of the ART centre}

Cleanliness must be guaranteed in accordance with documented sanitization protocols that take account of the recommendations of the competent authorities. Reference must be made to good clinical practice and the infection mitigation guidelines.

\section{Personnel and organization of the ART centre}

All the staff (medical, embryological, nursing, psychological support and secretarial) must receive training on the risks of COVID-19 infection and the safety measures to be taken. Specific instructions that can be modified according to the scientific knowledge that becomes available must be prepared and distributed.

Emergency plans for managing potential shortages of staff and equipment, and undesired exposure of the members of staff to the risk of COVID-19 infection must be elaborated. If the staff is sufficient in number, several rotating work groups should be organized to limit the spread of the virus in case of contagion in one group only.

As indicated in the Disaster Plan procedures in force, an agreement must be stipulated with another centre to guarantee continuity of care for the couples under treatment if the centre cannot complete a treatment cycle. Similarly, the emergency procedures should be revised or new procedures implemented, by finding equally competent doctors and embryologists from outside the centre to

- Scenario 1: patient and/or partner with negative triage or symptoms

- Scenario 2: patient and/or partner with positive triage or mild/aspecific symptoms

- Scenario 3: patient and/or partner with clear symptoms and/or positive SARS-COV-2 test

In presence of scenario $n .2$

It is recommended to provide rapid IgG-IgM and/or diagnostic workflow according to existing protocols in different regions or hospitals

Figure 2. Delineation of different scenarios after triage. 


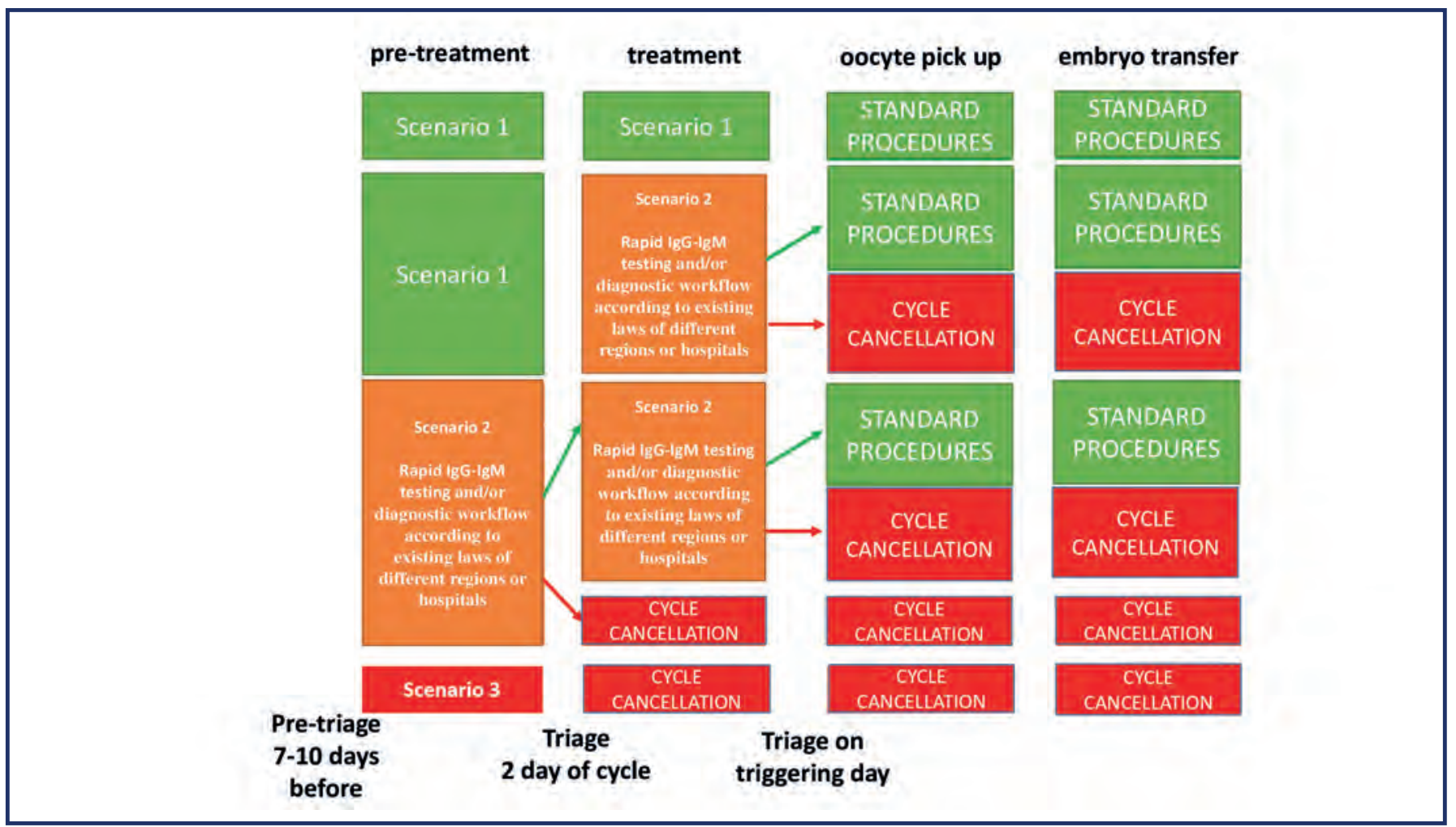

Figure 3. Summary flow chart for the management of ART treatments according to different scenarios.

replace the laboratory staff if the entire centre has to be put in quarantine.

\section{Access procedures}

The number of people present at the ART centre at the same time should be reduced to a minimum. The administrative staff must minimize contacts with patients and work behind screens when serving the public.

With a view to gradually resuming activities staggered in time, to avoid crowding at the single centres, priority of access will be given to couples with a diminished ovarian reserve, in accordance with the common classification systems.

Until the pandemic has been completely resolved, all health operators must wear the PPE conforming to the guidelines in force, where indicated.

The partner's presence must be limited to a minimum except for the day on which the sperm is to be donated. Nobody apart from the couple must be allowed into the centre.

The couple is requested to bring a face mask, alcohol hand sanitizers and / or gloves and shoe covers, which, if the couple has not managed to procure them, will be put at their disposal at the entrance to the ART centre.

When called to the centre on the telephone, the patients eligible to enter the facility for outpatient check-ups (ultrasound monitoring, semen analysis) must be informed of the time of the appointment by the offices concerned and the doctors involved. This time must be respected in order to avoid overlapping and the patients must be instructed to come alone, their state of health permitting, to avoid crowding in the waiting rooms and exam rooms; so, the staff must make sure the indications are followed.

There must be a limit to the number of people allowed in the waiting room, according to its size, respecting the distance of 1 meter and when this limit has been reached, any other patients present due to delays in the examinations will be requested to wait outside.

As a general rule, the number of operators present during the procedures must be kept to a minimum.

\section{Monitoring}

The doctor must wear gloves during ultrasound monitoring operations. The transvaginal ultrasound probe must be washed with a suitable disinfectant every time it is used. The use of ultrasound probe covers does not justify abandonment of the disinfection procedures to which the reusable devices must be subjected. The sheath must serve as a barrier. Compared to the normal probe sheaths, condoms are more effective (their acceptable qual- 
ity level is equivalent to that of surgical/medical gloves). The probe must be cleaned with a substance that does not damage it and that has a bactericidal, fungicidal and virucidal effect (for example, a product containing quaternary ammonium).

\section{Oocyte retrieval}

In the case of Scenario 1 or Scenario 2, with a negative rapid test result, a standard oocyte retrieval is performed. If the patient and/or partner is suspected to or actually tests positive, the cycle will be suspended.

If oocyte retrieval is inevitable, due to a risk of OHSS, the patient will be put on the "potentially COVID-19 positive patient pathway". This is a dedicated pathway, centred on observance of the general regulations and adoption of the strategies for access to the operating rooms, biocontainment and postoperative sanitization.

All the operators must therefore wear:

- shoe covers;

- first pair of gloves;

- disposable waterproof whitecoat;

- bouffant cap;

- FFP2 / FFP3 mask (according to the role);

- protective glasses/visor;

- second pair of gloves.

The operators must sanitize their hands, put on a disposable waterproof whitecoat, knee-high shoe covers and a bouffant cap, an FFP2 face mask and protective visor and two pairs of non-sterile gloves. At the end of the procedure, the material used must be deposited in the containers for infectious waste, and the stretcher, any other aids, the ultrasound scanner and aspirator must also be cleaned / disinfected.

The patient must enter the operating room directly with shoe covers, a disposable whitecoat, surgical mask and bouffant cap, washing his/her hands thoroughly with hydroalcoholic gel.

The preparation of the operating room must be organized using only the material strictly necessary for the type of operation to be performed and preferably using disposable material. All the material sused must be handled and disposed of in such a way as to prevent any form of contamination of the environment, the operators and the other patients. The team of doctors and biologists will put on the protective clothing before entering the operating room, in accordance with the standard procedure for a surgical operation, replacing the surgical mask with a FFP2 mask, glasses or protective visor and knee-high protective shoe covers.

Anaesthesiological procedures that limit the need for assisted ventilation are recommended, so as to reduce the risk of contagion for the operators to a minimum. In fact, some procedures may create spray and thus increase the transmission of the virus (tracheal intubation, non-invasive ventilation, etc.) and, in this case, an FFP3 mask must be worn. The entrance doors of the operating room must remain closed, thus reducing the movement of things and people to a minimum. During the surgical operation behind closed doors, the corridor will be sanitized for the first time and then subjected to a second in-depth sanitization procedure after the patient has been transferred to another room.

The oocytes retrieved and/or the embryos will be cryopreserved for subsequent use.

\section{Transfer}

For ascertained or strongly suspected COVID-19 positive patients (severe symptoms), the transfer should be deferred and the oocytes or embryos frozen, segmenting the ART cycle.

Only if the patient's negative result is certified by a diagnostic test (in accordance with the guidelines of the competent authorities) and the partner is negative, the transfer can be performed.

If the pre-FET triage form (administered at the start of supplementation with progesterone) reveals a "Scenario 3" (see "Access to the treatments"), the transfer should not be made. If it indicates a "Scenario 2 ", the choice will be made after a rapid test has been carried out.

Laboratory or preservation of embryos, oocytes and spermatozoa

To date, there is no information in the scientific literature on whether the virus is present in the human reproductive system, the follicular fluid or the seminal plasma or whether it adheres to oocytes, spermatozoa and embryos. This presence is improbable due to the lack of a receptor for the virus on these cells. For the fluids associated with them (seminal plasma and follicular fluid), no information is available so an adequate number of gamete washing should be performed, as laid down for the use of gametes of people affected by other viral diseases.

With regard to the ART procedures, there is currently no available information, on the risk of contamination of the culture systems with the SARS- 
COV-2 virus, or on the risk of interference with in-vitro embryo development. Studies on this subject are therefore necessary.

For the organization and implementation of the laboratory methods, the GISS intends to follow the recommendations of the Italian Embryology, Reproduction and Research Society (SIERR) (12). At the end of the lockdown period, all the staff at the centre (doctors, nurses, auxiliary staff) should receive training on topping up the cryocontainers in such a way as to preserve the cryopreserved material if the laboratory staff have to be put in quarantine. The cryobank management procedure should also be updated accordingly.

If electronic monitoring tools are not available, flexible procedures that minimize the presence of support staff without jeopardizing the efficacy of the process (e.g. other adequately equipped health operators or IT monitoring tools) should be elaborated. The laboratory environment and equipment must be sanitized with suitable detergents (quaternary ammonium solutions) at the end of every procedure or access to the environment.

If a treated patient is currently or subsequently found to be infected by COVID-19 (for example, cancer patients undergoing fertility preservation procedures), the laboratory spaces and the equipment (laminar flow hoods, incubators, etc.) used to handle the samples must be carefully decontaminated with a validated product; the manufacturers' recommendations, including dilution, contact time and safe handling, must be followed. This cleaning procedure must be performed after use and before the equipment is put back into normal service.

An extraordinary test of the alarm systems remote control should be planned.

\section{Cryopreservation}

To date there is no information about the risk of cross contamination between SARS-COV-2 infected samples and uninfected samples stored in the same container. If patients potentially at risk are treated, the samples should be cryopreserved in "high-safety" devices.

\section{CONFLICT OF INTERESTS}

The author declare that they have no conflict of interests. 


\section{REFERENCES}

1. https://www.asrm.org/globalassets/asrm/asrm-content/news-and-publications / covid-19/ covidtaskforce.pdf, 6th April 2020.

2. https: / / www.eshre.eu/Press-Room/ESHRE-News\#COVID19_April2, 6th April 2020.

3. Franchi M, Bosco M, Garzon S, et al. Management of obstetrics and gynaecological patients with COVID-19. Italian Journal of Gynaecology and Obstetrics 2020; 32(1):6-19. Doi: 10.36129/ jog.32.01.01.

4. https://www.cdc.gov/coronavirus/2019- ncov/ need-extra-precautions / pregnancy-breastfeeding.html, 6th April 2020.

5. https://www.gazzettaufficiale.it/atto/serie generale/ caricaDettaglioAtto/originario?atto. dataPubblicazioneGazzetta=2004-02-24\&atto. codiceRedazionale $=004$ G0062\&elenco30giorni $=-$ false, 6th April 2020.

6. https:// ourworldindata.org/age-structure, 6th April 2020.

7. https://www.sigo.it/comunicati-stampa/ covid-19-e-pma-a-rischio-8mila-trattamenti- al-mese-con-una-potenziale-perdita-di-natalita-mensile-di-circa-1500-bambini-per-i-massimi-esperti-riuniti-nel-giss-e-opportuno-avviare-nuovi-cicli-di-pma/,6th April 2020.

8. http://www.salute.gov.it/imgs/C_17_pubblicazioni_2866_allegato.pdf, 6th April 2020.

9. https://www.asrm.org/globalassets/asrm/asrm-content/news-and-publications/ covid-19/ covidtaskforceupdate1.pdf, 6 April 2020.

10. https://www.fertstertdialog.com/rooms/871covid-19/ posts / 64914-are-there-specific-patients-candidate-for-oocyte-collection-duringcovid-19-pandemic, 6th April 2020.

11. Vaiarelli A, Bulletti C, Cimadomo D, et al. COVID-19 and ART: the view of the Italian Society of Fertility and Sterility and Reproductive Medicine 2020 Apr 8th. Reprod Biomed Online. 2020;S1472-6483(20)30182-6. doi:10.1016/j. rbmo.2020.04.003.

12. De Santis L, Anastasi A, Cimadomo D, et al. COVID-19: the perspective of Italian embryologists managing the IVF laboratory in pandemic emergency. Hum Reprod 2020;35(4):1004-1005. doi:10.1093/humrep/deaa074. 